\title{
Ureteric sludge syndrome
}

\author{
R Nicholson, I Hewitt, A Kam
}

\begin{abstract}
Four cases of a form of obstructive uropathy previously unreported in children are described. All presented with oligoanuria and either flank pain or fluid retention and had evidence of crystalline sludge in their lower ureters. Three cases had an underlying crystalluria.
\end{abstract}

Currently children who present with oligoanuria after acute dehydrating or diarrhoeal illnesses are invariably assumed to have either prerenal azotaemia, acute tubular necrosis, haemolytic uraemic syndrome, or obstruction to drainage from a single kidney as might occur with calculus or ureterocoele. We present four cases of a form of obstructive uropathy, which may commonly be overlooked or misdiagnosed as one of the above conditions.

\section{Case reports}

CASE 1

A 13 year old boy presented in 1978 with acute abdominal pain, haematuria, and profuse uric acid crystalluria. His father had a history of urinary calculi, and his mother had recurrent abdominal pain associated with haematuria. On examination he was of normal stature with no renal enlargement or tenderness and his blood pressure was $120 / 80 \mathrm{~mm} \mathrm{Hg}$.

Serum creatinine concentration was $70 \mu \mathrm{mol} / 1$ and uric acid $0.33 \mathrm{mmol} / 1$. An intravenous urogram showed normal renal size and contour with good excretion bilaterally. A 24 hour urine sample confirmed an increased uric acid excretion of $4.4 \mathrm{mmol} /$ day (normal being less than $3 \cdot 5)$.

After discharge he remained well apart from non-specific abdominal pain. He represented four months later in acute renal failure. On admission he appeared uraemic and was mildly dehydrated. His blood pressure was $130 / 100$ $\mathrm{mm} \mathrm{Hg}$. The rest of the examination was normal. Serum creatinine was now $1550 \mu \mathrm{mol} / 1$ with serum uric acid of $1.7 \mathrm{mmol} / \mathrm{l}$. Haemoglobin concentration was $143 \mathrm{~g} / \mathrm{l}$; platelet count and blood film were both normal.

A presumptive diagnosis of acute urate nephropathy was made, and allopurinol started intravenously. After two haemodialyses he remained anuric and retrograde ureteric catheters were inserted. Crystalline sludge was noted at the base of the bladder, and urine samples from each ureter had high concentrations of uric acid. Both renal pelvices were washed out with an $8.4 \%$ solution of sodium bicarbonate. After this procedure there was a prompt diuresis of two litres of urine over the next hour. Without further dialysis renal function returned to normal.

CASE 2

In 1988 a 3 year old girl presented to a peripheral hospital over $1200 \mathrm{~km}$ north of Perth with a four day history of vomiting and diarrhoea. Urinalysis had shown large amounts of blood. Her condition deteriorated and she stopped passing urine. She developed a raised blood pressure to a maximum of $130 / 110 \mathrm{~mm}$ $\mathrm{Hg}$ with a rising serum creatinine concentration. A diagnosis of acute renal failure secondary to dehydration was made, and she was transferred by Royal Flying Doctor Service to our hospital. On admission she was unwell with facial oedema and raised blood pressure. Serum creatinine was $300 \mu \mathrm{mol} / \mathrm{l}$ and a full blood count was normal. Renal ultrasound showed swollen echogenic kidneys with dilated collecting systems and crystalline sludge (see figure). Twelve hours after admission she underwent a spontaneous diuresis and the ultrasound appearances had largely resolved by the next day. Urinalysis was not performed before discharge and therefore the nature of the sludge is not known. Because of the distance involved follow up was not possible.

\section{CASE 3}

A 14 month old boy presented in 1986 with a six day history of vomiting and diarrhoea. His urine output had been poor for 36 hours before admission. When initially seen he was $5 \%$

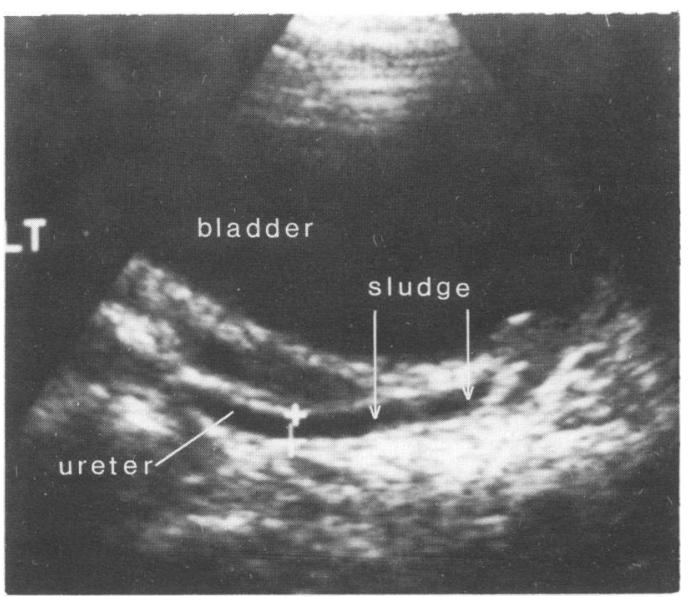

A renal ultrasound scan showing dilatation of the left ureter The picture shows debris in the distal end of the ureter (between the arrows) consistent with crystalline sludge. Note the absence of calculi and anatomical obstruction. 
dehydrated, his abdomen was soft and nontender, and a small sample of urine was heavily bloodstained. As he was refusing to drink, fluids were given initially by nasogastric tube; however, as he failed to improve and did not produce urine for over 48 hours intravenous hydration was started on day three. He quickly developed periorbital and scrotal oedema with a raised blood pressure of $180 / 100 \mathrm{~mm} \mathrm{Hg}$. Serum creatinine was $245 \mu \mathrm{mol} / \mathrm{l}$. Haemoglobin, blood film, and platelet count were normal. Renal ultrasound showed normal renal parenchyma, dilated collecting systems, and an empty bladder. The dilatation appeared acute with no evidence of caliceal clubbing. The likely diagnosis was considered to be vesicoureteric junction obstruction secondary to crystal sludge.

He remained irritable with a raised blood pressure for 24 hours, despite several doses of hydralazine and frusemide intravenously. A spontaneous diuresis occurred on day four with an improvement in his condition and a return to normal blood pressure. Repeat ultrasound one month after discharge showed a normal collecting system on the right and mild dilatation on the left. There was no debris evident. A micturating cystourethrogram showed no abnormality.

Subsequent studies on the child showed a raised cystine excretion at $195 \mathrm{mmol} / \mathrm{l}$ suggesting heterozygous cystinuria. Both his parents also had cystinuria in the heterozygous range.

\section{CASE 4}

A 13 year old boy, who received regular transfusions for $\beta$ thalassaemia, presented in 1981; he was unwell with a fever of $39.5^{\circ} \mathrm{C}$, sore throat, and cough. He was thought to have a viral illness, was treated symptomatically, and his regular blood transfusion was delayed.

He deteriorated progressively with increasing abdominal pain, vomiting, and diarrhoea and was readmitted three days later. At this stage he was passing only a few drops of heavily bloodstained urine. He was mildly dehydrated and afebrile, with loin tenderness on the left side. His haemoglobin was $86 \mathrm{~g} / \mathrm{l}$, white cell count $13.0 \times 10^{9} / 1$ with a normal differential. Blood film and platelet count were normal. Serum creatinine concentration was $395 \mu \mathrm{mol} / \mathrm{l}$ and uric acid $0.11 \mathrm{mmol} / \mathrm{l}$. Urine and blood cultures taken at that time later proved to be sterile.

His urine output remained poor, and on the fourth day he underwent bilateral renal exploration and insertion of nephrostomy drainage tubes. At operation the right kidney was swollen, discoloured, and oedematous; old blood clot was washed from the ureter. The findings on the left side were similar but less noticeable; urine, but no clot, was found under some pressure in the pelvis. Urine output improved and renal function returned to normal after irrigation.

Subsquent studies showed that his uric acid excretion was abnormally high while on desferrioxamine with an excretion of $9 \mathrm{mmol} /$ day. This fell to within the normal range when this treatment was discontinued.

\section{Discussion}

All the children described exhibit features one would expect in an obstructive uropathynamely, bilateral dilatation of the ureters associated with impaired renal function, prompt diuresis, and return of outflow tract calibre to normal once the obstruction is relieved. We demonstrated blood or crystalline sludge in dilated lower ureters. There was no evidence of stones, uterocoeles, or other obstructive elements.

Evidence that crystalline sludge was the cause of the obstruction is clear from the first two cases as it was seen directly on cystoscopy in the former and on ultrasound in the latter. The presence of sludge is strongly implied in the latter two cases, and they both demonstrated raised excretion of either cystine or uric acid. The boy with thalassaemia had gross haematuria and blood clots were found at operation. Though it is possible that the clot was the primary cause of the obstruction, it must be exceedingly rare for gross haematuria to cause a bilateral ureteric obstruction. It would be reasonable to suggest that the bleeding and renal oedema were secondary to the obstruction that had been present for at least four days before surgery.

Predisposing factors that we have identified as a cause of the syndrome are firstly dehydration, which in all of our cases followed either a febrile or diarrhoeal illness, and secondly an underlying crystalluria, which was demonstrated in three out of four cases. Two had a raised excretion of uric acid and one of cystine.

Tubular obstruction due to crystalluria has previously been described with sulphonamides, ${ }^{1}$ ethylene glycol poisoning, primary hyperoxaluria, ${ }^{2}$ and uric acid nephropathy complicating the treatment of leukaemia and lymphomas. ${ }^{3}$ Our first case was initially thought to have uric acid nephropathy; however, the failure to respond to dialysis and the prompt recovery after retrograde irrigation makes this diagnosis unlikely. To the best of our knowledge ureteric obstruction due to crystalluria has not previously been reported in children. It has been mentioned briefly in one adult textbook but no details were given. ${ }^{4}$

This is an important condition to recognise as failure to do so could lead to acute renal failure or severe hypertension as occurred in cases 1 and 3 respectively. The diagnosis should be considered when a child presents with dehydration and oligoanuria, particularly when there is failure to respond to adequate volume replacement. Ultrasound examination of the vesicoureteric junction is likely to assist the diagnosis. It has been established previously that ultrasound is a highly effective method of screening for obstructive causes of renal failure ${ }^{5}$ and in evaluating renal colic. ${ }^{6}$ In our series cases 1 and 4 did not have renal ultrasound examinations as this facility was not available at our hospital until 1982. In cases 2 and 3 however, this procedure made a significant contribution to the diagnosis.

Treatment should initially consist of intravenous fluid boluses and diuretics. Although two of our children had spontaneous diuresis, the 
other two required surgical decompression with either an antegrade or retrograde ureteric irrigation. We would therefore advise that failure to respond to conservative management within 24 hours, or the development of hypertension, should lead to more direct attempts to relieve obstruction.

Finally we recommended that after recovery, all of these children should be investigated for the possibility of an underlying crystalluria.

1 Arneil GC. Twenty-nine children with sulphonamide haematuria. Lancet 1958;i:826-7.
2 Barratt TM. Acute renal failure. In: Holliday M, Barratt TM, Vernier R, eds. Pediatric nephrology. 2nd Ed. Baltimore: Williams and Wilkins, 1987:766-72.

3 Kjellstrand CM, Campbell DC, Von Hartitzsch B, et al. Hyperuricaemic acute renal failure. Arch Intern Med 1974; 133:349-59.

4 Coe FL, Favus MJ. Nephrolithiasis. In: Braunwald E, Isselbacher KJ, Petersdorf EG, et al, eds. Harrison's principles of intermal medicine. 11th Ed. New York: McGraw Hill, 1987:1211-5.

5 Curatola G, Mazzitelli R, Monzani G, et al. The value of ultrasound as a screening procedure for urological disorders in renal failure. $\mathcal{F}$ Urol 1983;130:8-10.

6 Erwin BC, Carroll BA, Sommer FG. Renal colic: the role of ultrasound in initial evaluation. Radiology 1984;152: 147-50.

\title{
Pain and measles, mumps, and rubella vaccination
}

\author{
Ronan Lyons, Fenton Howell
}

\begin{abstract}
Seventy seven children (mean age $\mathbf{4 4 . 2}$ months) were entered in a randomised double blind study to find out if two commonly used measles, mumps, and rubella (MMR) vaccines (Pluserix and MMR II) caused pain on injection. Pain was judged by the amount of crying. Infants given MMR II were 2.31 times more likely to cry than those given Pluserix.
\end{abstract}

A vaccination programme to eliminate measles, mumps, and rubella (MMR) from the British Isles was introduced in October 1988. Two vaccines, Pluserix (Smith Kline French) and MMR II (Wellcome), have been used extensively in the campaign and they have comparable immunogenicity and reactogenicity. ${ }^{1}$ Since the start of the campaign some family doctors in Ireland reported that the MMR II vaccine caused more distress to children than the Pluserix vaccine. Similar reports came from the United Kingdom. ${ }^{2}$ Although the data sheet for MMR II states that the vaccine may cause burning or stinging at the injection site for a short time because the reconstituted vaccine is slightly acid (pH 6.2-6.6), we know of no published evidence to suggest that immediate pain at the injection site is a problem. ${ }^{3}$ We decided to investigate the matter further.

\section{Subjects and methods}

Eastern Health Board, Community Care Area 7, Aras Daimhin, Croke Park, Jones's Road, Dublin 3, Republic of Ireland Ronan Lyons Fenton Howell

Correspondence to: Dr Howell.

Accepted 16 October 1990

(ArchDisChild 1991;66:346-7)
An inner city coeducational preschool population from a socially deprived area with a poor uptake of the MMR vaccine was chosen for the study. All children who had not been vaccinated were invited to attend for vaccination on one of two consecutive days. All children being vaccinated were accompanied by someone they knew well. Each child received $0.5 \mathrm{ml}$ of vaccine subcutaneously through a 25 gauge needle into the deltoid region of the left arm. All vaccines were given by a single experienced doctor (RL). Crying after the injection was used as a measure of pain, and was assessed by the other doctor (FH). A randomised double blind study design was used, in which each vaccine was randomly selected from a previously coded lot and neither the doctors nor the recipients were aware of which vaccine had been used until the trial had been completed. Vaccinated children were kept separate from unvaccinated ones to avoid 'contamination'. Data were analysed by $\chi^{2}$ test, Fisher's exact test, or Student's $t$ test.

\section{Results}

Of the 97 children presenting, 20 cried before vaccination. Of the remaining 77 (37 boys and 40 girls), 37 (mean (SD) age $43 \cdot 4(11 \cdot 1)$ months) received Pluserix and 40 (mean (SD) age $45 \cdot 2$ (10.7) months) received MMR II. There were no differences in the age between the groups or sex of the children.

The table shows the number that cried after vaccination. Children who received MMR II were 2.31 times more likely to cry than those who received Pluserix ( $95 \%$ confidence interval $1 \cdot 16$ to $4 \cdot 60$ ). Neither age nor sex were significantly associated with crying. Of the 28 children who cried after vaccination, none cried for longer than a minute.

\section{Discussion}

The results of this study suggest that pain is a real and unnecessary side effect of some MMR vaccines. Though we recognise that there are many difficulties in measuring pain, we feel that crying after vaccination is a reasonable measure

Incidence of crying after the two vaccines

\begin{tabular}{lclc}
\hline & $\begin{array}{l}\text { No who } \\
\text { cried }\end{array}$ & $\begin{array}{l}\text { No who } \\
\text { did not cry }\end{array}$ & Total \\
\hline $\begin{array}{l}\text { Pluserix } \\
\text { MMR II }\end{array}$ & 8 & 29 & 37 \\
\hline Total & 20 & 20 & 40 \\
\hline
\end{tabular}

